\title{
UMA CONSISTENTE METODOLOGIA PARA ACOPLAMENTO DE SIMULAÇÕES 1D-3D APLICADO AO SISTEMA DE HVAC DE UM VEICULO PARA RESOLUÇÃO DO CICLO DE AR-CONDICIONADO E CABINE INTERNA
}

\author{
Joel Sanvezzo Junior \\ Siemens Industry Software Ltda. \\ Email: joel.sanvezzo@siemens.com
}

\section{RESUMO}

A abordagem multidisciplinar, que envolve diferentes tipos de formulação na resolução de problemas de engenharia, vem sendo cada vez mais requisitado na indústria para se encontrar um equilíbrio entre a qualidade dos resultados e o tempo computacional. Neste trabalho é apresentado uma abordagem de acoplamento entre soluções 1D e 3D aplicados para o sistema de HVAC de um automóvel, de maneira a ser resolvido o ciclo de refrigeração do sistema de ar condicionado e a cabine. Em uma primeira etapa do artigo é apresentado o modelo 1D para resolução do ciclo de refrigeração do sistema de ar-condicionado utilizando o software Simcenter Amesim. Em uma segunda etapa, é apresentado o modelo 3D para a resolução da cabine através da aplicação de CFD (Computational Fluids Dynamics) no software Simcenter STAR-CCM+. Por fim, tem-se a descrição do acoplamento entre estes modelos via cosimulação. Através deste acoplamento, tem-se uma completa caracterização do sistema de HVAC, a nível de componentes e subcomponentes pelo modelo $1 \mathrm{D}$, e a resolução do comportamento do fluxo de ar no interior da cabine, pelo modelo 3D. Além do conforto térmico dos ocupantes do veículo, este tipo de análise fornece informações importantes a respeito do comportamento físico e energético do sistema, principalmente durante etapas conceituais em projetos de engenharia.

\section{INTRODUÇÃO}

Com a crescente preocupação pelo aumento de eficiência energética nos produtos, aumenta-se a procura por ferramentas que auxiliam a tomada de decisões de maneira rápida e com os custos reduzidos. Neste sentido, as aplicações envolvendo CAE (Computer Aided Engineering) são cada vez mais utilizadas na busca por cenários mais eficientes, uma vez que com a construção de modelos computacionais, é possível minimizar os testes físicos e o tempo de desenvolvimento do produto. No mercado automotivo, diversas áreas têm mostrado esforços nesta direção, entre elas a de gerenciamento térmico, que possibilita a investigação de uma grande diversidade de fenômenos que contribuem para a redução do consumo de combustível e a emissão de gases tóxicos. Tais problemas de natureza térmica são originados basicamente 
da circulação e passagem de fluidos e sua transferência de calor conjugada no interior de sistemas e compartimentos. Neste sentido, o sistema de HVAC (Heat, Ventilation and Air Conditioning) de um automóvel representa uma grande área de atuação, visto as interações que ocorrem no nível sistêmico e sua interação com a cabine do veículo. Para uma robusta e diversificada caracterização do problema, salienta-se a aplicação de ferramentas multidisciplinares e multifisicas, como encontradas em softwares 1D e 3D, que utilizadas de maneira isolada ou acoplada, atuam na previsão de fenômenos e detecção de problemas, principalmente em etapas conceituais de projeto. Através de modelos 3D, tem-se uma resolução detalhada (ponto-a-ponto) do domínio de interesse e geralmente é aplicado para se obter a resolução do comportamento interno e externo de componentes "chaves". Por outro lado, modelos 1D trazem informações importantes em nível de sistema como na obtenção de eficiências de componentes e curvas características. Estas duas abordagens de simulação podem ser vistas como complementares, e o seu acoplamento na resolução de problemas está sendo cada vez mais procurado uma vez que minimiza o tempo computacional e evita a imposição de condições de contornos aproximadas quando ocorre a resolução dos problemas de maneira separada.

Chen et al. [1] descreveram uma abordagem de acoplamento 1D-3D para simular o comportamento térmico em aeronaves. Através do modelo 1D foi resolvido o sistema de controle ambiental (ECS-Aircraft Environmental Control System) e pelo modelo 3D-CFD, foi resolvido o escoamento no interior da aeronave. $\mathrm{O}$ acoplamento entre os códigos é realizado via script escrito na linguagem de programação C. Os autores salientam diversas vantagens com este tipo de abordagem, entre elas a trocas de informações no mesmo passo de tempo, que elimina a necessidade de uma prescrição de condição de contorno obtida de maneira empírica.

Kumar et. al [2] descreveram um modelo de acoplamento 1D-3D para se avaliar o comportamento térmico conjugado no compartimento do motor. Através do modelo 1D foi resolvido o circuito de lubrificação do bloco do motor e a temperatura de alguns corpos sólidos. $\mathrm{O}$ modelo $3 \mathrm{D}$ foi utilizado para resolver o comportamento térmico e fluidodinâmico do escoamento no interior do vão motor. Como conclusão, os autores destacam a possibilidade de se avaliar diferentes ciclos de direção e a utilização de condições de contornos interativas e acopladas.

Karamjit et al. [3] descreveram uma modelagem 3D para previsão do comportamento de Warmup e Cool Down utilizando a técnica de CFD. O objetivo principal da análise foi de garantir o conforto térmico dos ocupantes através da previsão do fluxo de ar e temperatura. $\mathrm{O}$ trabalho foi validado experimentalmente para diferentes velocidades do veículo em uma análise transiente, onde a diferença máxima entre os valores medidos e simulados foi de $5^{\circ} \mathrm{C}$.

No presente trabalho, é apresentado uma metodologia de acoplamento entre ferramentas 1D e 3D para se modelar o sistema de HVAC de um automóvel de maneira a ser resolvido o ciclo de refrigeração do sistema de ar condicionado e o mapeamento térmico detalhado da cabine do automóvel. Através da modelagem 1D, tem-se a caracterização completa do ciclo de refrigeração do sistema do ar-condicionado, com a presença de todos os componentes e etapas do sistema em regime transiente. Pelo modelo 3D, tem-se a solução diferencial do volume interno da cabine aplicando a técnica de CFD, trazendo informações pontuais e refinadas a 
respeito do mapeamento térmico. O acoplamento entre os modelos é de duas vias em um processo de co-simulação, ou seja, as informações são trocadas simultaneamente entre os softwares em um regime transiente. Tal abordagem é também conhecida como acoplamento forte [4], no qual se tem uma maior qualidade dos resultados devido a troca de informações simultâneas em cada passo de tempo.

\section{MODELAGEM 1-D DO SISTEMA DE AR CONDICIONADO}

O sistema de refrigeração do ar condicionado é modelado de maneira unidimensional utilizando o software Simcenter Amesim. Os componentes do sistema são incluídos na análise, como o condensador, compressor, evaporador e tubo curto. A Fig. 1(a) mostra de maneira simplificada um esquema do ciclo de refrigeração adotado. No ciclo, o compressor fornece a vazão de refrigerante pelo sistema, na qual também ocorre o aumento da pressão de vapor. No condensador, ocorre a liberação de energia do refrigerante para a mistura de ar, e o estado físico do refrigerante é alterado de vapor para um fluxo bifásico. Com a passagem no orifício de tubo curto, a pressão diminui e como resultado, tem-se uma suave diminuição da temperatura do fluido. Após esta etapa, o fluido ao passar pelo evaporador, começa a evaporar. No sistema, adiciona-se um acumulador com objetivo de assegurar que apenas vapor superaquecido adentre no compressor. A Fig. 1(b) ilustra uma representação teórica do ciclo de refrigeração do gás R134a, onde é possível observar todas as etapas do ciclo, sendo etapa 1 para compressão, etapa 2 para condensação de vapor, etapa 3 para expansão e etapa 4 de evaporação. Através desta curva é possível avaliar critérios importantes do ciclo como na previsão dos efeitos de superaquecimento e sub-resfiramento. Através deste tipo de modelo é possível projetar e analisar o sistema do ciclo de refrigeração incluindo as etapas de stop e start do compressor, conforme situação real de operação.

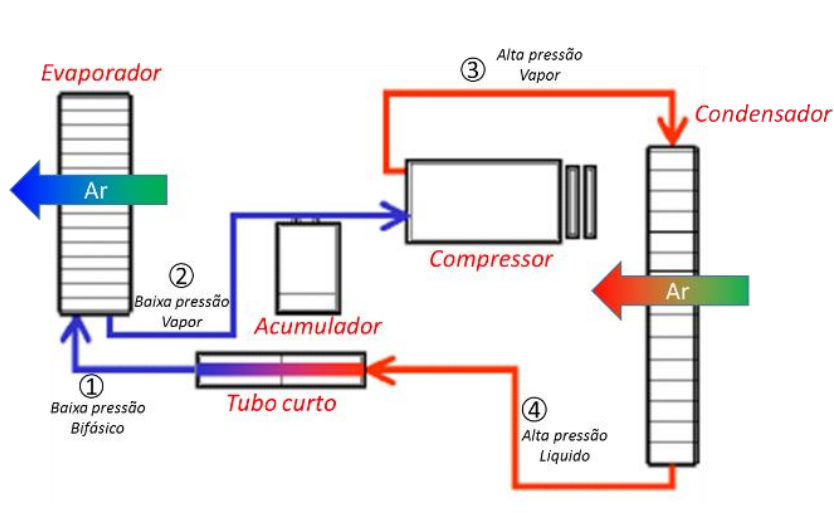

(a)

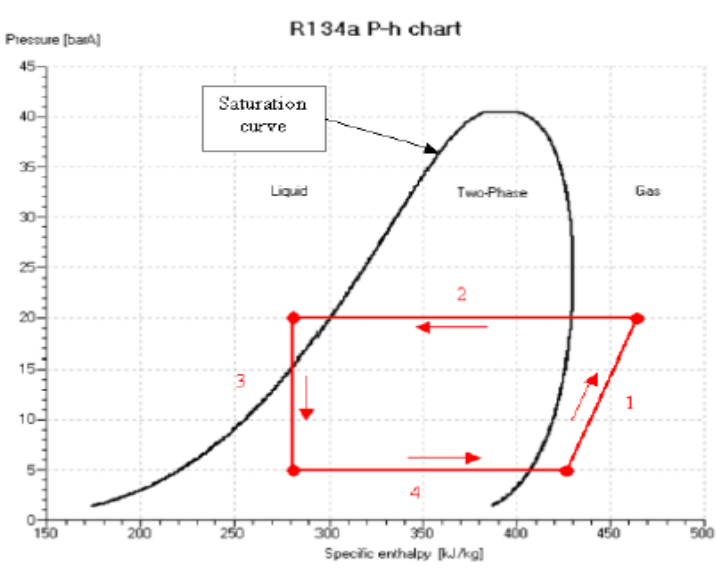

(b)

Figura 1. (a) Esquema do Sistema de refrigeração e (b) representação teórica do ciclo P-h.

O software Simcenter Amesim tem a capacidade de promover bibliotecas prontas para estes tipos de analises [5]. Para construir um modelo completo de um sistema de ar condicionado, são necessárias três bibliotecas: biblioteca térmica (TH), biblioteca de fluxo bifásico (TPF) e biblioteca de ar condicionado (AC). A biblioteca TH é usada para modelar os trocadores de calor e também a mistura de ar úmido ambiente. A biblioteca TPF é composta de elementos 
básicos (condições de contorno, sensores, dutos, perdas de pressão e componentes para modelar o fluxo interno do refrigerante. A biblioteca AC é composta por componentes globais específicos do sistema AC. Desta maneira, se tem uma rápida e efetiva avaliação das condições de trabalho do sistema e do rendimento, assim como para o design de cada componente.

No presente trabalho, são fornecidos para o modelo 1D as informações de entrada do ar como temperatura, pressão, vazão e humidade e também as informações operacionais do compressor. Como resultado, tem-se a resolução de todas as etapas do ciclo e as informações na saída do evaporador e que são utilizadas como dados de entrada para a resolução 3D. O ciclo completo utilizado no presente trabalho é mostrado na Fig. 2(a) onde é possível observar todos os componentes do sistema. O fluxo de ar húmido no evaporador, assim como no condensador, tem as seguintes características: pressão de $1 \mathrm{~atm}$, temperatura de $30^{\circ} \mathrm{C}$ e humidade relativa de $40 \%$. A vazão de ar húmido que é insuflado para o evaporador é variável conforme condições a seguir: vazão de $0.11 \mathrm{~kg} / \mathrm{s}$ para os primeiros $150 \mathrm{~s}$, vazão de $0.02 \mathrm{~kg} / \mathrm{s}$ no período de $150 \mathrm{a}$ $350 \mathrm{~s}$ e $0.11 \mathrm{~kg} / \mathrm{s}$ para os últimos 100s. Este comportamento visa capturar o efeito de desligamento da tomada de ar do ar-condicionado pelo motorista, de maneira a estar sincronizado com o desligamento do compressor. Com relação ao compressor, foram admitidos dois níveis de rotação, de carga máxima $(2500 \mathrm{rpm})$ e carga mínima (100 rpm), conforme os seguintes períodos de tempo (Fig.2(b)): 0-150s rotação de $2500 \mathrm{rpm}$; 150-350s rotação de 100 rpm; 350-450s rotação de $2500 \mathrm{rpm}$. Este comportamento de acionamento do compressor é geralmente descrito por um ciclo de condução e o presente trabalho busca a aplicação de um ciclo simples de on-off. Há atualmente diversos tipos de ciclo de condução, produzidos por diferentes países e organizações para avaliar o desempenho dos veículos.

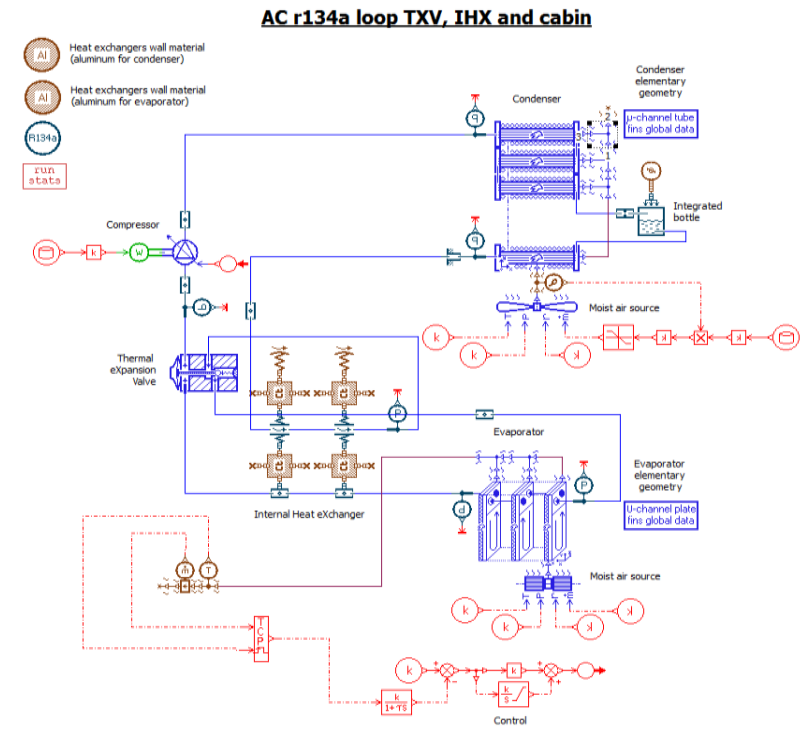

(a)

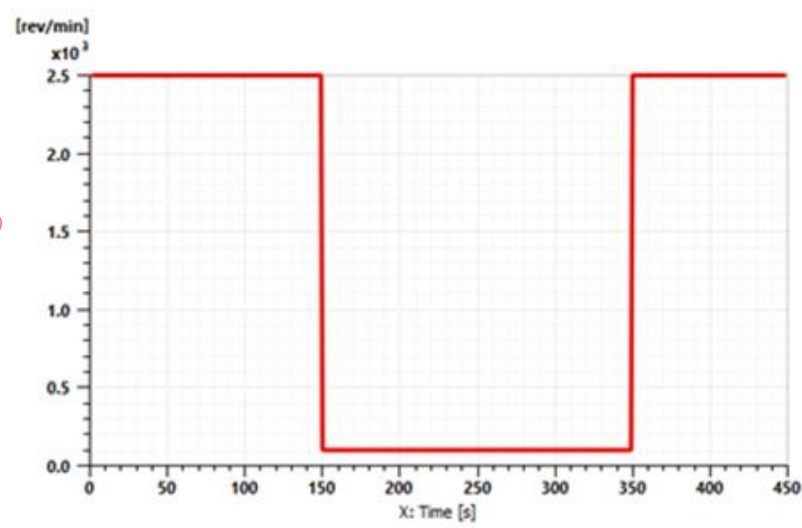

(b)

Figura 2. (a) Esquema do ciclo de refrigeração 1D e (b) ciclo de direção do compressor.

Pela Fig.2 (a), observam-se diversos componentes e subsistema no ciclo que podem ser objetos de estudos em uma análise mais aprofundada. Curvas características, eficiências, design geométricos e comportamentos físicos podem ser explorados, auxiliando o desenvolvimento de projetos voltados para o sistema de HVAC de um automóvel. O presente trabalho objetiva o 
controle de temperatura da cabine pelo ajuste de rotação do compressor e assim, análises mais profundas acerca de cada componente não são contempladas.

\section{MODELAGEM 3D DA CABINE INTERNA}

Modelos CFD tridimensionais são comumente utilizados para se calcular o comportamento de fluidos no interior ou exterior de compartimentos, como é o caso da cabine interna do automóvel. Com esta técnica, basicamente se tem a resolução pontual do volume interno da cabine através da solução das equações de Navier-Stokes. Aplicando-se o modelo RANS (Reynolds Averaged Navier-Stokes) de turbulência nas equações de Navier-Stokes [4] encontrase uma forma geral para as equações governantes, conforme Eq. 1 mostrada abaixo:

$$
\frac{\partial(\rho \Phi)}{\partial t}+\operatorname{div}(\rho u \Phi)=\operatorname{div}(\Gamma \operatorname{grad} \Phi)+S
$$

sendo, $\rho$ a densidade volumétrica, $t$ o tempo, $u$ a velocidade do fluxo, $\Gamma$ o coeficiente de difusão, $S$ o termo fonte e $\Phi$ uma variável geral. A Eq.1 se torna a equação da conservação da massa quando $\Phi$ representa a pressão, equação de conservação de momentum quando $\Phi$ representa a velocidade e equação de conservação de energia quando $\Phi$ representa temperatura.

Neste trabalho, tomou-se como referência a cabine interna do modelo Freelander fornecido pela Jaguar Land Rover [3], conforme mostrado na Fig.3. Pela Fig.3 (c) é possível identificar as entradas no duto de HVAC, que por sua vez se comunica com o sistema de ar condicionado localizado no vão motor do veículo. Este duto apresenta uma entrada de ar na seção inferior e duas saídas, uma para o fluxo central e outra para o fluxo lateral.

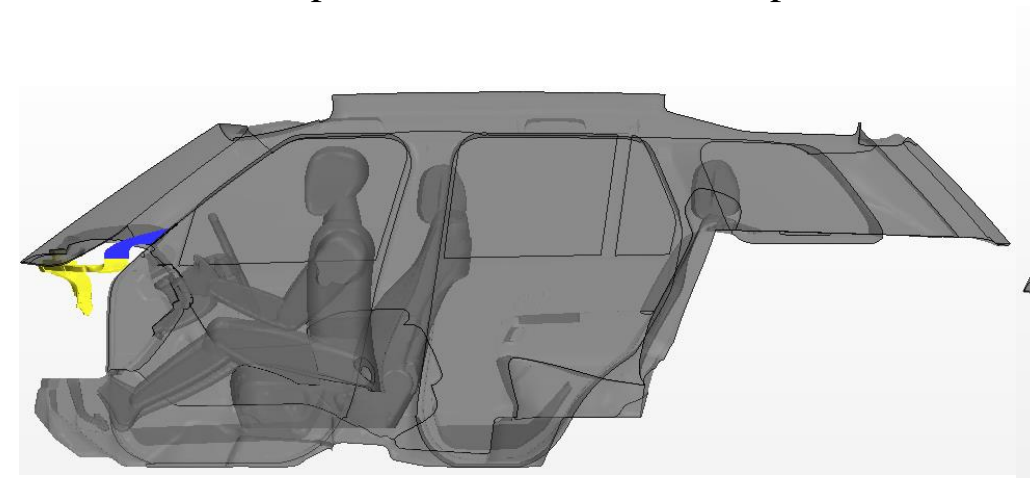

(a)

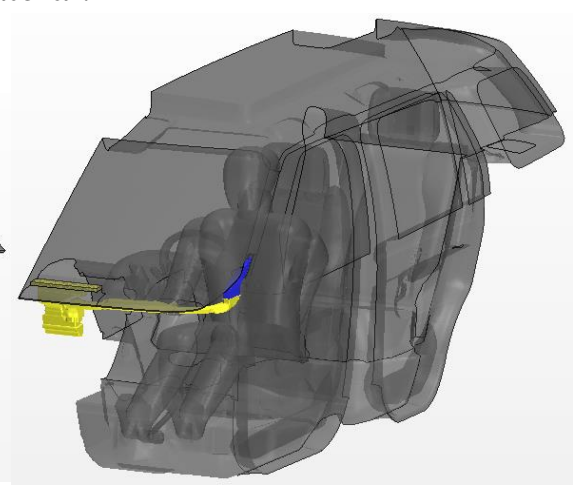

(b)

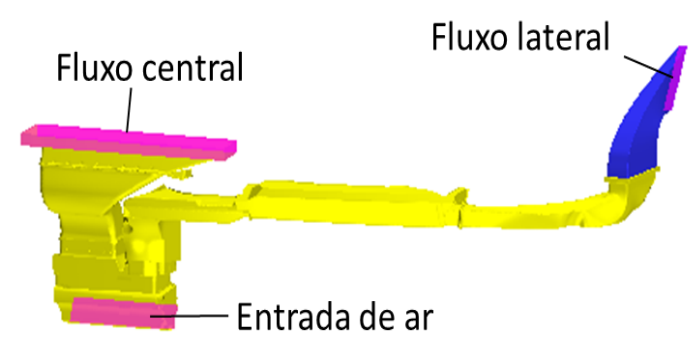

(c)

Figura 3. (a) Vista lateral e (b) isométrica do volume interno da cabine para análise CFD e o (c) duto de ventilação do sistema de ar-condicionado. 
Após a saída nos dutos, o fluxo de ar resfriado é encaminhado para o interior da cabine. Através da aplicação de CFD, tem-se a resolução do campo de velocidade e temperatura em todo domínio interno (dutos e cabine) e que são resolvidos conforme condições de contornos impostas nas fronteiras. São definidas quatro condições de contorno: entrada, saída, simetria e térmica. A condição de entrada está conforme Fig.3(c), sendo que a saída se localiza na porção inferior da cabine. A condição de simetria foi aplicada na seção média do veículo de forma a se simplificar o cálculo computacional. A condição térmica de convecção foi aplicada nos vidros laterais, para-brisa e teto, de maneira a se incluir um efeito convectivo com o ambiente externo. O presente modelo CFD foi construído no software Simcenter STAR-CCM+, onde as condições na entrada do duto são fornecidas pelo modelo 1D provido pelo software Simcenter Amesim. A Fig. 4 ilustra a malha computacional da cabine interna e dos dutos de ventilação, onde é possível se observar um refinamento médio. O modelo apresenta malha poliédrica e camadas de prismas para capturar os efeitos fluidodinâmicos e térmicos próximos das paredes, totalizando aproximadamente 1,5 milhões de células.

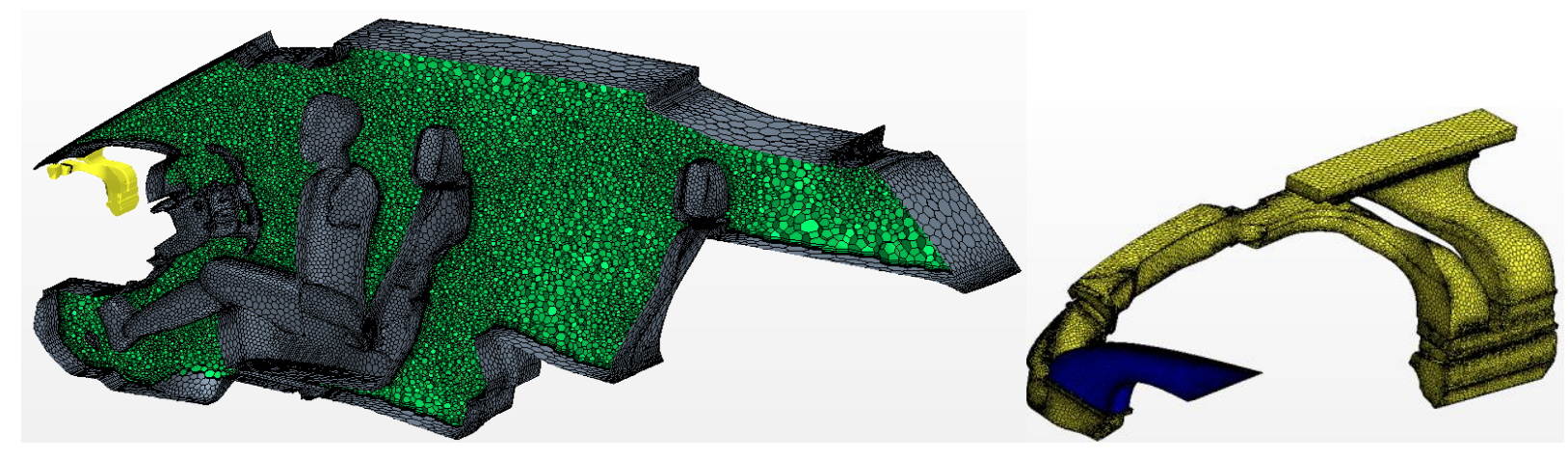

Figura 4. (a) Malha computacional da cabine interna e (b) dos dutos de ventilação.

\section{MODELO INTEGRADO 1D-3D}

A capacidade de realizar co-simulações em softwares comerciais denota um importante avanço na área de simulações CAE devido à capacidade de se resolver modelos de maneira interativa. $\mathrm{O}$ acoplamento entre softwares 1D e 3D oferece diversas vantagens no ciclo de desenvolvimento de produtos. Através deste acoplamento, elimina-se a necessidade de prescrição de condições de contorno empíricas, uma vez que tais informações são fornecidas instantaneamente durante a solução. Outro fator importante é de serem explorados os aspectos positivos de cada modelagem. Por exemplo, a resolução de sistemas multidisciplinares e seus componentes por simulações 3D, como no caso de um sistema de refrigeração, seria impraticavelmente grande. Com isso, os modelos 1D são preferíveis nos casos em que se deseja uma modelagem completa no nível do sistema. Por outro lado, os modelos 3D são preferíveis quando os efeitos tridimensionais são significativos, como é o caso da cabine interna e dos dutos de HVAC. Portanto, a presente metodologia denota um forte acoplamento entre os modelos, trazendo resultados mais rápidos e com alta qualidade.

Um fluxograma do procedimento é mostrado na Fig. 5, onde observa-se a chamada dos modelos e as trocas de informações no passo de tempo, $\Delta$ t. Durante o primeiro passo de tempo, o código Simcenter Star-CCM + lê a primeira solução que vem do Simcenter Amesim e resolve o modelo 
3D. Depois disso, o solver Simcenter Amesim é chamado pelo Simcenter Star-CCM + e a solução 1D é realizada auxiliada pelos resultados 3D que foram resolvidos anteriormente. No lado $1 \mathrm{D}$, um novo intervalo de tempo atual, $\Delta \mathrm{t}+1$, é resolvido e ocorre uma nova troca de informações com o modelo 3D. Este laço continua até que a solução acoplada satisfaça o tempo total da simulação.

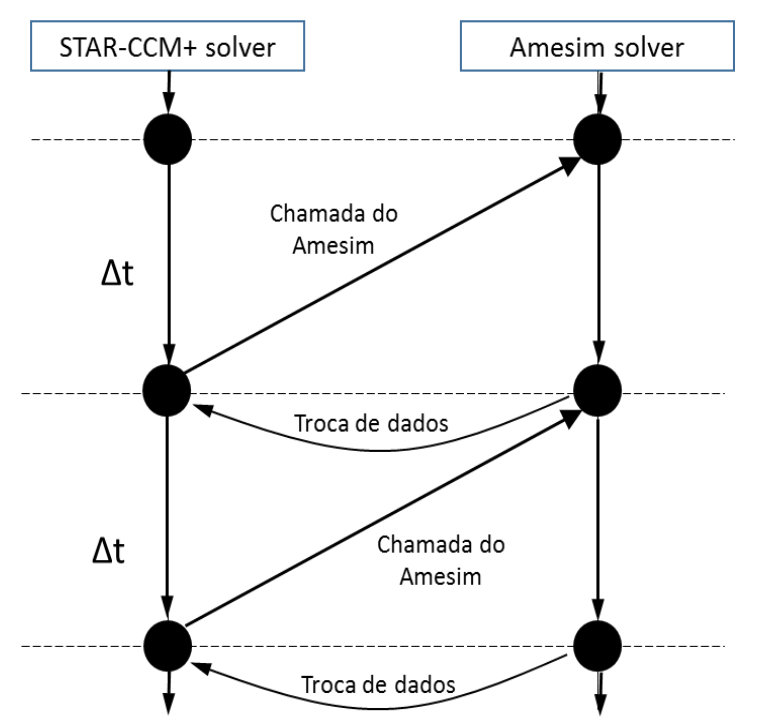

Figura 5. Troca de informações interativas entre os códigos Star-CCM+e Amesim.

No presente trabalho é realizado uma co-simulação entre os softwares Simcenter STAR-CCM+ e Simcenter Amesim. A comunicação entre eles ocorre através de soquetes TCP/IP usando um elemento TCP (elemento dynamic_cosim_socket). Um elemento TCP (Fig. 6) contém portas de entrada e saída, que trocam dados com o STAR-CCM +. As portas no lado esquerdo do elemento enviam dados para o STAR-CCM +, enquanto as portas no lado direito do elemento recebem dados do STAR-CCM +. O modelo 3D aplica os campos importados do Amesim nas fronteiras do modelo CFD, como no caso da entrada dos dutos.

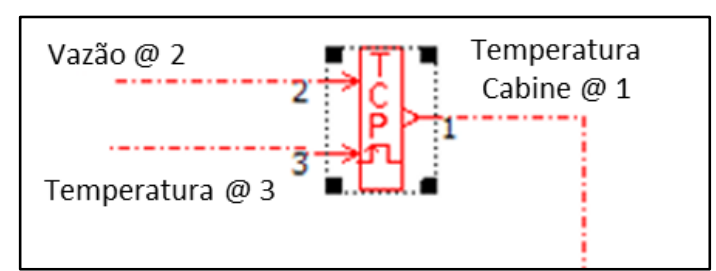

(a)

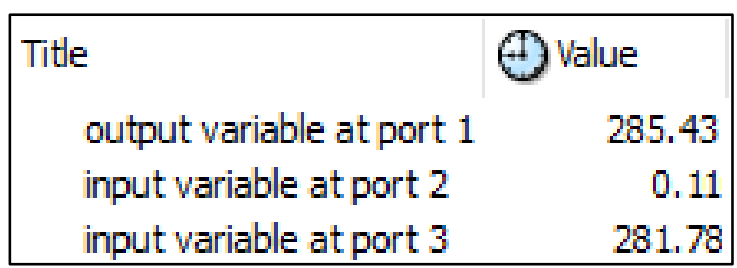

(b)

Figura 6. (a) Elemento TCP no Amesim para troca de informações com o Star-CCM+e (b) exemplo das variáveis em cada porta.

Objetivando o conforto térmico na cabine interna do veículo, avalia-se o campo de temperatura em um plano médio da cabine de maneira a verificar o comportamento da solução no regime transiente. A escolha do plano médio para análise da temperatura serve como exemplo de aplicação, no entanto, outras localidades e regiões poderiam ser objeto de estudo uma vez que a solução 3D-CFD fornece os resultados ponto-a-ponto do escoamento em toda geometria. Por exemplo, regiões específicas no corpo dos ocupantes e regiões de "hot spots" no interior da cabine são facilmente identificáveis com este tipo de análise. 
Conforme mencionado anteriormente, a temperatura inicial do ar na cabine, assim como para o escoamento de ar no evaporador, é de $30^{\circ} \mathrm{C}$. O passo de tempo, $\Delta \mathrm{t}$, em ambos os modelos é de 0.1 segundos para garantir a sincronia entre as rodadas. O tempo total de simulação é de 450s. Após o início da simulação, tem-se a resolução do acoplamento em três etapas. Na primeira etapa, a rotação no compressor é mantida constante em um valor nominal (2500 rpm) por um período de 150s, ocorrendo assim, a diminuição da temperatura do ar que é encaminhado para o duto de ventilação. Esta temperatura é encaminhada para o modelo CFD, onde é resolvido o campo de temperatura da cabine no mesmo passo de tempo. Como resposta do modelo CFD, tem-se a temperatura média do plano de corte escolhido. Esta temperatura não é utilizada para realimentar o sistema 1D devido critério de simplificação deste trabalho, porém salienta-se a possibilidade de realização deste tipo de comunicação. Esta primeira etapa do ciclo ocorre até que seja atingido o regime permanente no interior da cabine. Após este período, aplica-se uma diminuição da rotação do compressor $(100 \mathrm{rpm})$ por um período de $200 \mathrm{~s}$, de maneira a se simular o efeito de desligamento do ar-condicionado. Esta ação leva a um aumento na temperatura do ar e, consequentemente, da cabine interna. Na terceira e última etapa do sistema, é retomada a rotação nominal do compressor por um período de 100 s, finalizando-se a análise.

\section{RESULTADOS E DISCUSSOES}

A distribuição de temperatura ao longo do tempo no plano médio da cabine é mostrada na Fig.7. Este plano foi escolhido por estar próximo ao ocupante e fornece informações a respeito do comportamento térmico e fluidodinâmico nesta posição.

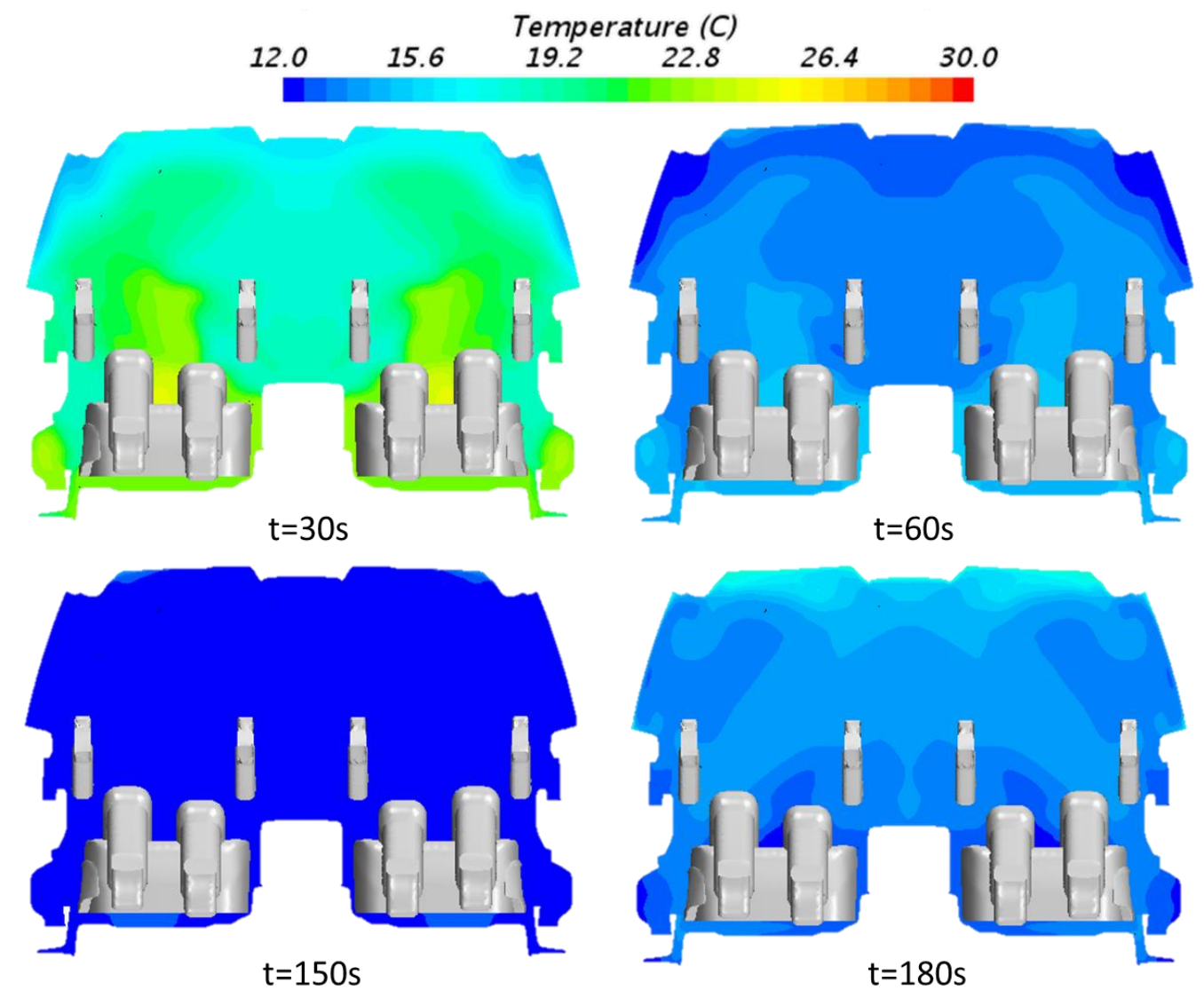




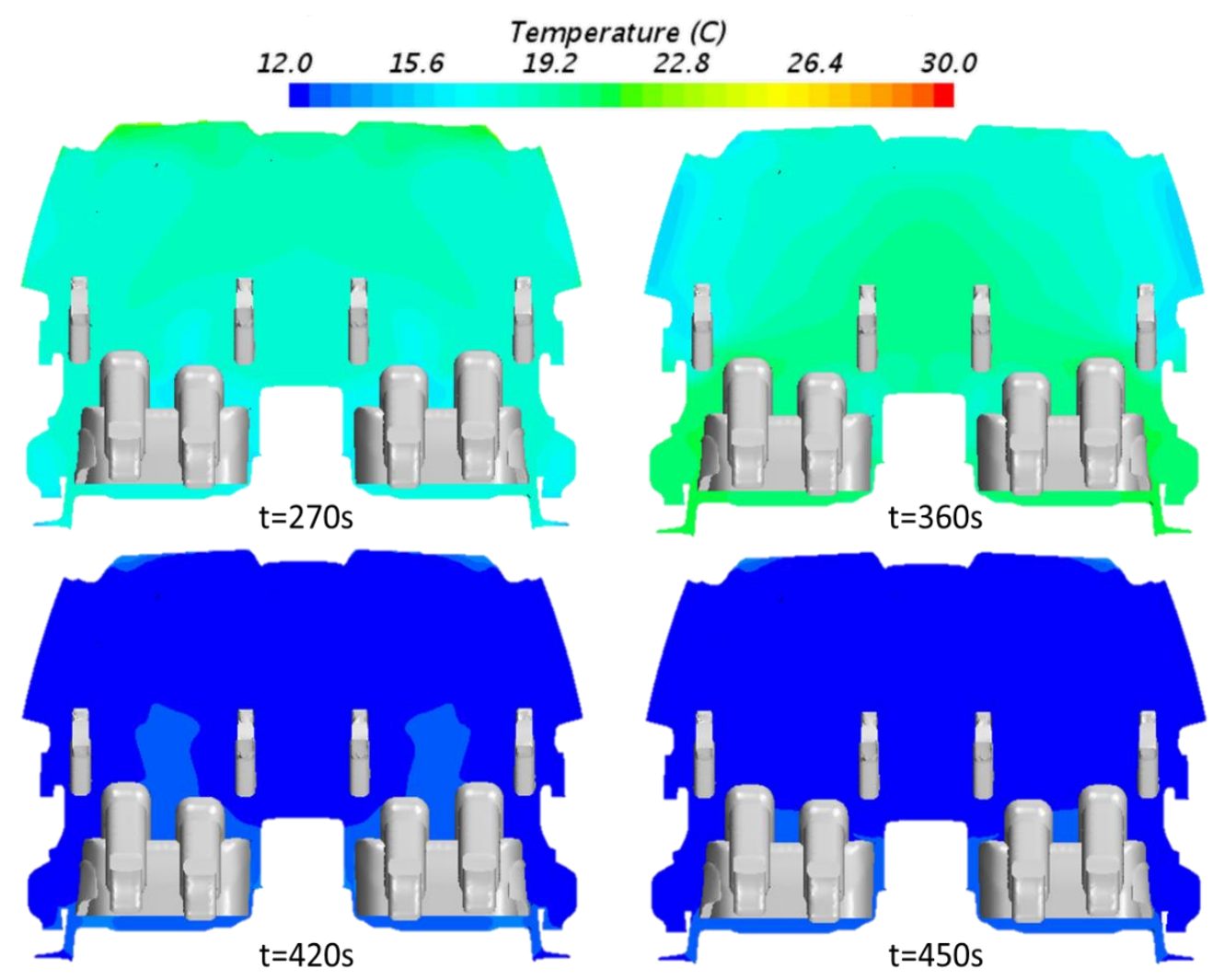

Figura 7. Distribuição de temperatura no plano médio da cabine.s

Pela Fig.7 é possível observar as três etapas da simulação, sendo de acionamento do compressor (0-150s), decaimento da vazão do compressor (150-350s) e re-acionamento do compressor (350-450s).

De posse do comportamento térmico em todos os pontos, é possível identificar regiões críticas que diminuem o conforto térmico dos ocupantes e assim, propor melhorias que se adequem as características do projeto.

Pela Fig. 8 tem-se os comportamentos do ciclo de direção prescrito no compressor e da temperatura média no plano de referência da cabine durante o tempo total de simulação. Notase o comportamento sincronizado e inversamente proporcional entre essas grandezas. Para a carga máxima do compressor (2500 rpm), tem-se uma diminuição da temperatura na cabine até que seja atingido o regime permanente. Após o desligamento, em 150s, tem-se uma diminuição da rotação do compressor e, consequentemente, um aumento da temperatura média da cabine. Este efeito é decorrente da diminuição da vazão mássica de fluido refrigerante pelo compressor, o que totaliza uma menor troca térmica no condensador e evaporador. Na etapa final, tem-se o re-acionamento nominal do compressor, na qual se atinge novamente a temperatura média mínima no interior da cabine. Este ciclo apresenta um comportamento simples de on-off, porém pode ser facilmente modificado para ciclos padrões utilizados pela indústria. Além disso, a Fig. 8 também mostra o período de "cool-down", que é realizável durante os primeiros 50 segundos. Este período é uma medida importante do desempenho do veículo, uma vez que garante o tempo necessário para se atingir o objetivo de refrigeração. 
A Fig.9 mostra a evolução dos trocadores de calor e da potência provida para o refrigerante pelo compressor. Observa-se o comportamento sincronizado dos componentes do ciclo de refrigeração (evaporador, condensador e compressor) com as etapas do ciclo de direção. As taxas de calor no condensador e evaporador decrescem substancialmente com a diminuição da rotação do compressor, devido a diminuição da vazão mássica de refrigerante que é bombeado no sistema. Outra relação importante que pode ser extraída da Fig. 9 é do coeficiente de performance, $C O P$, que é definido como a razão entre o calor entregue pelo evaporador pela potência mecânica do compressor. No regime estacionário do presente trabalho, tem-se um valor de $C O P$ de 3,15. Além do $C O P$, diversos parâmetros e variáveis pertinentes ao projeto de refrigeração podem ser explorados na busca por cenários mais eficientes.

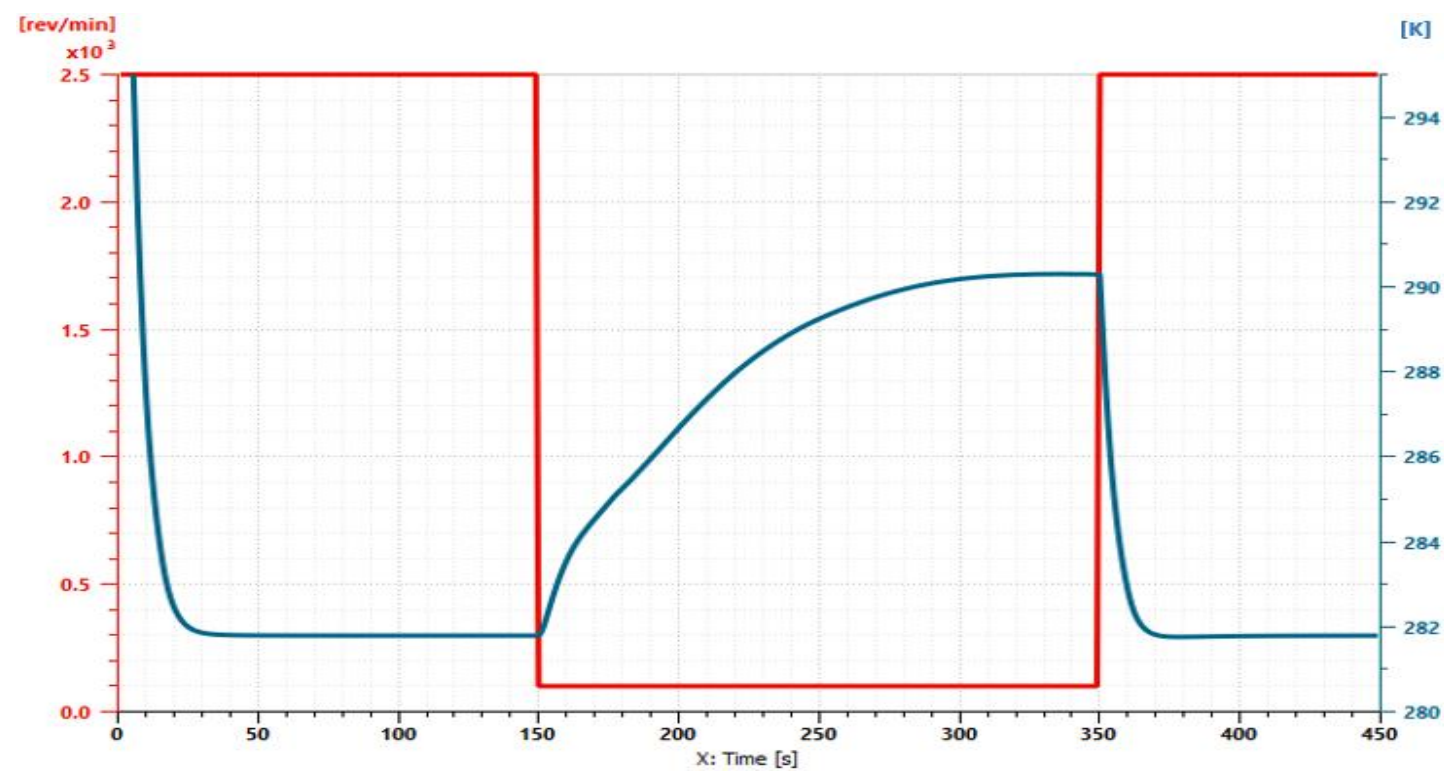

Figura 8. Curvas de rotação do compressor conforme ciclo de direção (vermelho) e temperatura média no plano de referência da cabine (azul).

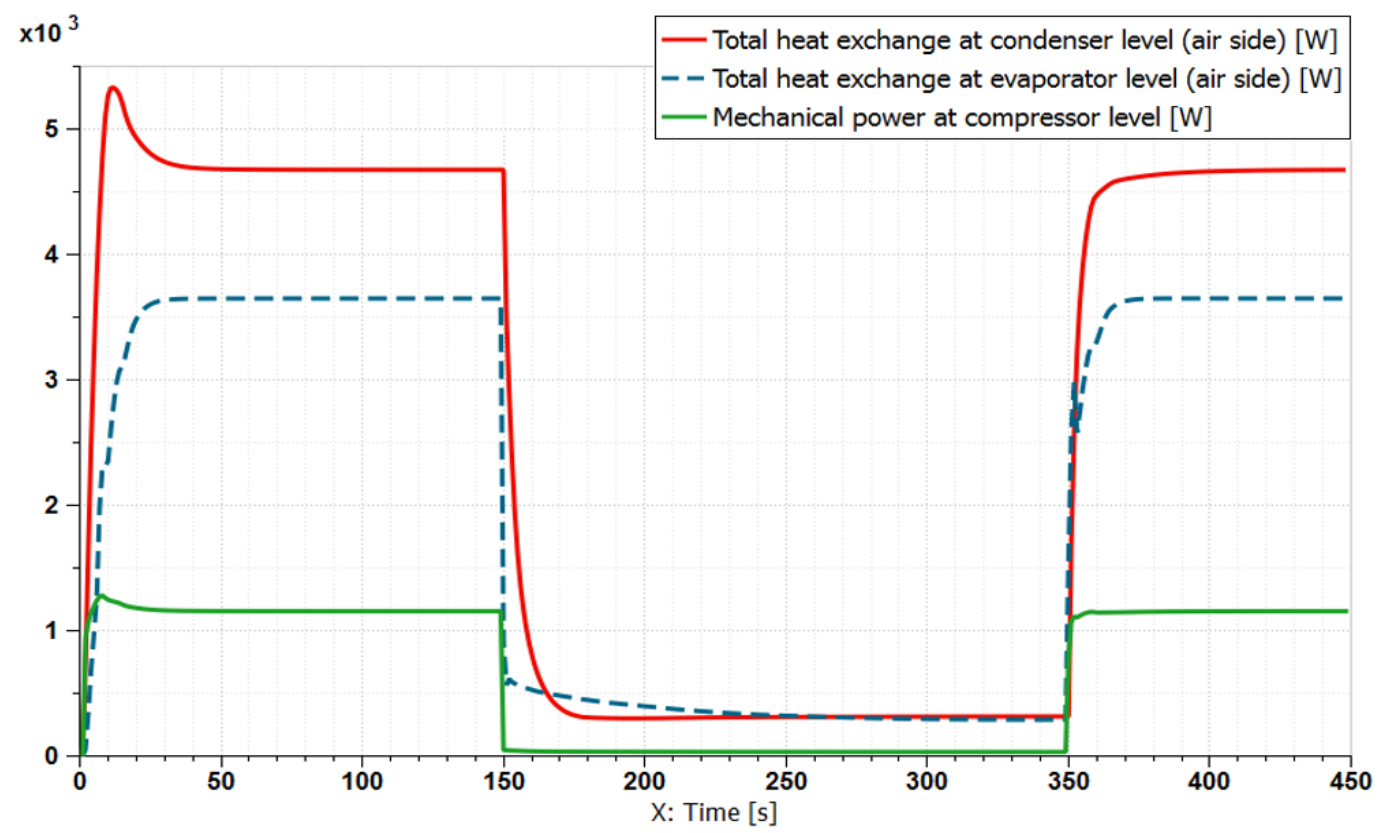

Figura 9. Curvas dos trocadores de calor e compressor para o ciclo de refrigeração. 
A Fig.10 mostra o ciclo de refrigeração do presente modelo no diagrama pressão x entalpia. É interessante observar a diferença com o ciclo padrão do refrigerante e identificar as etapas onde as ineficiências são geradas, como nas regiões de sub-resfriamento e superaquecimento. O subresfriamento do fluido que deixa o condensador é uma prática comum, pois garante que o fluido que entra na válvula de expansão esteja totalmente no estado líquido. O superaquecimento do vapor que deixa o evaporador evita que gotículas de líquido adentrem o compressor. Mesmo com alguns fatores positivos, estas regiões devem ser minimizadas de maneira a se aumentar a eficiência dos processos. Através desta curva também podem ser levantadas outras análises de eficiências dos processos como, por exemplo, para o COP, eficiência termodinâmica e volumétrica, entre outros.

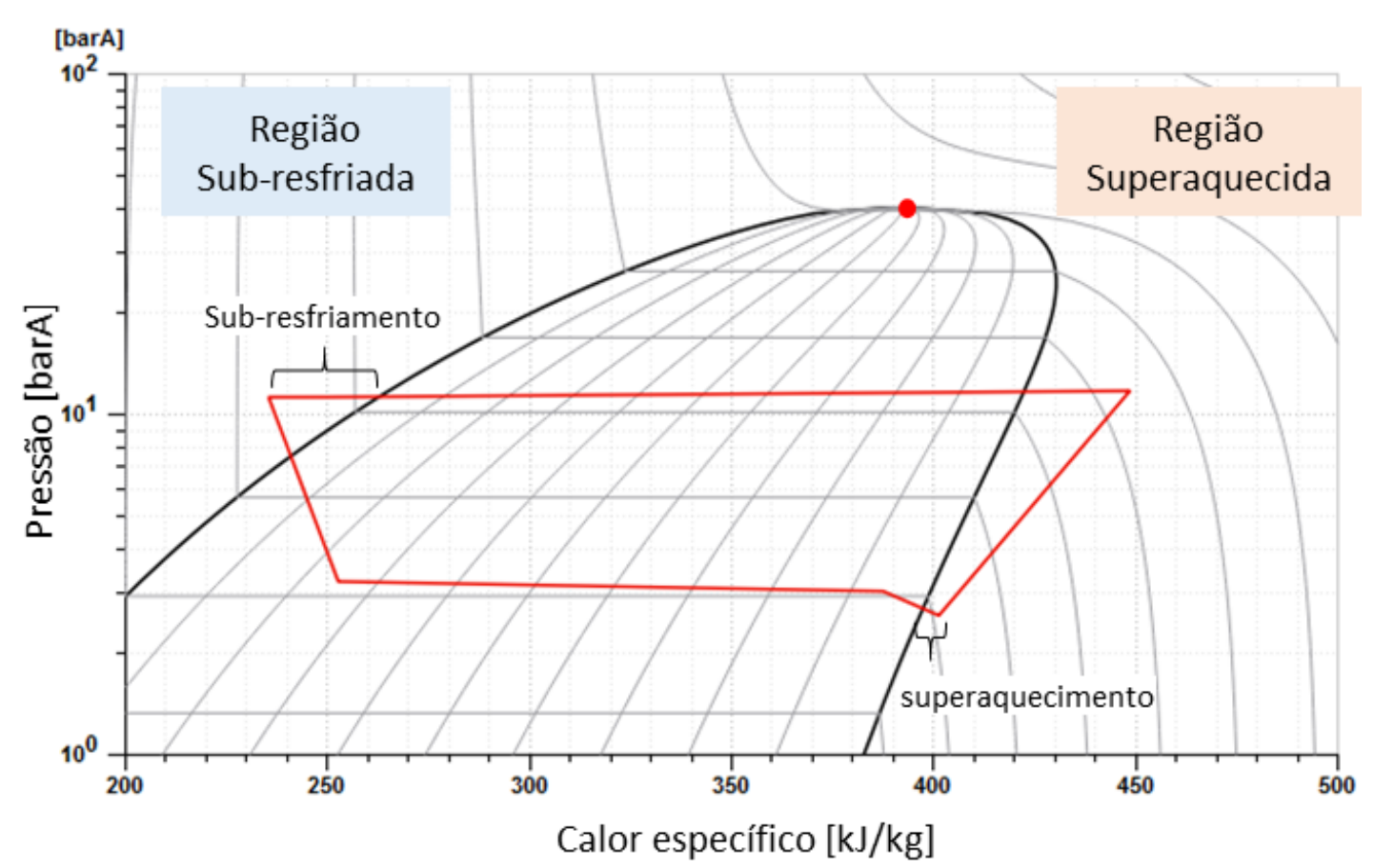

Figura 10. Diagrama P-h atual do ciclo de refrigeração.

\section{CONCLUSÃO}

Este trabalho apresentou uma metodologia de acoplamento 1D/3D utilizando os softwares Simcenter STAR-CCM+ e Simcenter Amesim, aplicados ao sistema de HVAC de um automóvel para os ciclos de refrigeração e resolução da cabine interna. O objetivo foi de se verificar o conforto térmico dos passageiros alterando-se o funcionamento do compressor em um ciclo especifico de condução. De posse dos resultados, observa-se o comportamento físico de ambos modelos, 1D e 3D, a respeito de informações importantes como do perfil de temperatura da cabine e curvas de eficiências do sistema. Tais informações são de extrema importância durante etapas conceituas de engenharia, na qual serão analisadas e estimadas as demandas energéticas do projeto. Neste sentido, demonstra-se como a aplicação de metodologias CAE são uma poderosa ferramenta para se obter cenários cada vez mais eficientes e com menor tempo de projeto. Além disso, destaca-se a facilidade de uso da metodologia de 
co-simulação utilizando os softwares Simcenter Amesim e Simcenter Star-CCM +, uma vez que não há necessidade de implementação de códigos externos visto que a comunicação via interface gráfica.

A geometria utilizada fornecida pela JLR, assim como do sistema de refrigeração, serviu como exemplo de aplicação e os resultados apresentados não se comprometem com a realidade do produto, devido diferenças pertinentes ao sistema de refrigeração e com as condições de contornos aplicadas. No entanto, o objetivo principal do trabalho, de descrever uma metodologia de acoplamento entre os modelos, foi atingida. Maiores investigações acerca dos resultados e comportamentos devem ser avaliadas para uma melhor caracterização do problema.

Como trabalho futuro, sugere-se a realimentação do sistema 1D através da resolução do sistema 3D. Pode ser realizado, por exemplo, a leitura da temperatura média ou pontual da cabine em locais específicos para se calibrar o acionamento automático do sistema de refrigeração. Outra analise futura se refere a exploração de design de componentes específicos como dos dutos de ventilação e dos designs dos trocadores de calor, que podem ser explorados de maneira lógica e automatizada na busca por melhores famílias de resultados. Além das variáveis e analises mencionadas, é possível encontrar diversos parâmetros que podem ser objetos específicos de estudos e assim, encontrar componentes otimizados sob o ponto de vista de eficiência energética.

\section{REFERÊNCIAS BIBLIOGRÁFICAS}

[1] Xiyuan, CHEN; Jianzhong, YANG; Tianpeng, YU, Shibin YANG. A Coupled 1D/3D CoSimulation Approach in Simulating Aircraft Cabin Temperature Field. Tianjin Key Laboratory of Civil Aircraft Airworthiness and Maintenance, Civil Aviation University of China, Tianjin, China, 2016.

[2] Kumar, Saket; Pascual, Daniel. Unsteady Thermal Management Simulations for a Passenger Vehicle using 1D and 3D Tools. M.Sc Thesis, Department of Applied Mechanics CHALMERS UNIVERSITY OF TECHNOLOGY Gothenburg, Sweden, 2016.

[3] Sandhu, Karamjit. Simulation of the Interior Cabin Warm-up and Cool Down using CFD. STAR Global Conference, 2014.

[4] H. Versteeg; W. Malalasekera; An Introduction to Computational Fluid Dynamics: The Finite Volume Method, 2nd Edition. Loughborough University, 2007.

[5] Simcenter Amesim User's Guide. Cooling System Library, 2016.

\section{AGRADECIMENTOS}

$\mathrm{O}$ autor reconhece a geometria da cabine fornecida pela Jaguar Land Rover e as pessoas diretamente envolvidas com o artigo mencionado "Simulation of the Interior Cabin Warm-up and Cool Down using CFD” [3], Dr. Karamjit Sandhu da JLR e Mark Farrall da Siemens. No 
presente estudo, um ciclo de refrigeração padrão e condições de contorno simplificadas foram escolhidos pelo autor para ilustrar o processo. 